\title{
Diagnostic Signs of Accommodative Insufficiency
}

\author{
PILAR CACHO, OD, ÁNGEL GARCÍA, OD, FRANCISCO LARA, OD, and MA MAR SEGUÍ, OD \\ Departamento Interuniversitario de Óptica, Universidad de Alicante, Spain (PC, AG, MMS), Departamento de Oftalmología, AP y ORL, \\ Universidad de Murcia, Spain (FL)
}

\begin{abstract}
Purpose. To determine which are the most sensitive tests, together with accommodative amplitude, to classify accommodative insufficiency (AI), we analyzed the relation between monocular estimated method (MEM) dynamic retinoscopy, monocular and binocular accommodative facility (MAF, BAF), and positive relative accommodation (PRA) with or without the presence of reduced amplitude of accommodation. Methods. We studied 328 symptomatic patients who presented consecutively to an optometric clinic. From this sample, we selected the 41 patients who presented amplitude of accommodation at least $2 \mathrm{D}$ below the minimum age-appropriate amplitude according to Hofstetter's formula: $15-0.25 \times$ age. We also selected data from 40 consecutive subjects (control group) with no general binocular disorders and normal accommodative amplitudes. We studied the specificity and sensitivity of the four signs related with the accommodative insufficiency: high MEM dynamic retinoscopy, failing MAF and BAF with minus lenses of \pm 2 D flipper lenses, and low PRA. Results. Using the standard deviation as the cutoff, the specificity values were $\mathrm{MEM}=\mathbf{0 . 8 8}, \mathrm{MAF}=1, \mathrm{BAF}=0.93$, and $\mathrm{PRA}=1$. When using the mean value as the cutoff, the specificity diminished, fundamentally for MEM. The sensitivity for the 41 patients using standard deviation as the cutoff was MEM $=0.44, \mathrm{MAF}=0.34, \mathrm{BAF}=0.27$, and PRA $=0.27$, and when using the mean value as the cutoff the four, sensitivity values increased. Conclusions. According to the sensitivity results, with both cutoffs used, failing the $\pm 2 \mathrm{D}$ MAF test seems to be the sign that is most associated with the accommodative insufficiency. (Optom Vis Sci 2002; 79:614-620)
\end{abstract}

Key Words: accommodative insufficiency, accommodative amplitude, monocular estimate method dynamic retinoscopy, monocular and binocular accommodative facility, positive relative accommodation

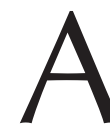
ccording to the literature, accommodative insufficiency is an accommodative anomaly characterized fundamentally as having an amplitude of accommodation consistently below the appropriate age level. However, several authors who have studied this anomaly refer to different diagnostic criteria. In Table 1, several studies have used different criteria to make the diagnosis. As can be observed, there are two major criteria for diagnosing accommodative insufficiency: by means of a single clinical sign and by using multiple signs.

According to the reports that refer to this accommodative disorder using single clinical signs, some of them define accommodative insufficiency simply as diminished accommodative amplitude. This is the case for Daum, ${ }^{1}$ who uses the criterion of having $2 \mathrm{D}$ below Hosfstetter' ${ }^{2}$ minimum age-amplitude formula, $15-0.25$ $X$ age, to establish a patient with a low amplitude of accommodation. However, not all of the studies use Hofstetter's formula. Other authors, such as Matsuo and Ohtsuki, ${ }^{3}$ apply the sign of reduced accommodative amplitude according to Duane's $s^{4}$ criterion, although they do not specify exactly how many diopters below. Similarly, Russell and Wick ${ }^{5}$ define accommodative insuf- ficiency as when patients exhibit an accommodative amplitude at least $2.5 \mathrm{D}$ below that expected for their respective age, based on Duane's age norms. ${ }^{4}$ Other authors, however, define accommodative insufficiency considering Hofstetter's formula, but binocularly. For example, Dwyer and Wick ${ }^{6}$ define accommodative insufficiency as when the binocular accommodative amplitude is $2 \mathrm{D}$ or more below the expected value.

A number of authors have used an additional clinical sign, added to the essential one of having diminished accommodative amplitude. Among them are the studies of Hokoda ${ }^{7}$ and Rouse et al. ${ }^{8}$ In Hokoda's ${ }^{7}$ report, accommodative insufficiency is diagnosed when the patient has a push-up monocular accommodative amplitude at least $2 \mathrm{D}$ below Hofstetter's calculation for minimum age-appropriate amplitude and positive relative accommodation (PRA) $\leq 1.25$ D. However, Rouse et al. ${ }^{8}$ classified patients with accommodative insufficiency (associated with convergence insufficiency) when the subject failed Hofstetter's minimum amplitude formula or had greater than a $+1 \mathrm{D}$ lag on monocular estimate method dynamic retinoscopy.

The other group of studies of accommodative insufficiency re- 
TABLE 1.

Summary of several studies reporting different criteria for diagnosing accommodative insufficiency (Al).

\begin{tabular}{|c|c|}
\hline Authors & Criteria Used For Diagnosing Al \\
\hline Daum $^{1}$ & $\mathrm{AA}^{a} 2 \mathrm{D}$ below Hofstetter ${ }^{2}$ minimum amplitude formula: $15-0.25 \times$ age \\
\hline Matsuo and Ohtsuki ${ }^{3}$ & Reduced AA based on Duane's criterion ${ }^{4}$ \\
\hline Russell and Wick ${ }^{5}$ & AA at least $2.5 \mathrm{D}$ below the expected for age based on Duane's criterion \\
\hline Dwyer and Wick ${ }^{6}$ & Binocular AA 2 D or more below the expected for the patient's age using Hofstetter's formula \\
\hline Hokoda $^{7}$ & $\begin{array}{l}\text { AA at least } 2 \mathrm{D} \text { below Hofstetter's minimum amplitude formula } \\
\text { Decreased PRA, } \leq 1.25 \mathrm{D}\end{array}$ \\
\hline Rouse et $\mathrm{al}^{8}$ & AA fails Hofstetter's minimum amplitude formula, or MEM retinoscopy $>+1.00 \mathrm{D}$ \\
\hline Scheiman et al $^{9}$ & $\begin{array}{l}\text { AA }>2 \text { D from mean for age using Hofstetter's formula } \\
\text { At least } 2 \text { signs of } 4 \text { additional signs }{ }^{b}\end{array}$ \\
\hline Porcar and Martínez-Palomera ${ }^{10}$ & $\begin{array}{l}\text { AA at least } 2 \mathrm{D} \text { below Hofstetter's minimum amplitude formula } \\
\text { PRA decreased, } \leq 1.25 \mathrm{D} \\
\text { Failing accommodative facility with }-2.00 \mathrm{D} \text {, monocular } \leq 6 \mathrm{cpm} \text {, binocular } \leq 3 \mathrm{cpm} \\
\text { High MEM, } \geq 0.75 \mathrm{D} \\
\text { High fused cross-cylinder, } \geq 1.00 \mathrm{D}\end{array}$ \\
\hline Lara et $\mathrm{al}^{11}$ & $\begin{array}{l}\mathrm{AA} \text { at least } 2 \mathrm{D} \text { below Hofstetter's minimum amplitude formula } \\
\text { Fail monocular accommodative facility with }-2.00 \mathrm{D}, \leq 6 \mathrm{cpm} \\
2 \text { Signs of } 3 \text { additional signs }{ }^{c}\end{array}$ \\
\hline
\end{tabular}

${ }^{a}$ AA, accommodative amplitude; PRA, positive relative accommodation; MEM, monocular estimated method.

${ }^{b}$ Scheiman et $\mathrm{al}^{9}$ : See text for description of these four additional signs.

${ }^{c}$ Lara et $\mathrm{al}^{11}$ : See text for description of these three additional signs.

fers to multiple clinical signs for diagnosing this accommodative anomaly. Scheiman et al..$^{9}$ specified that it was necessary to have an accommodative amplitude $2 \mathrm{D}$ or less than the minimum established by Hofstetter's formula and to exhibit two of the following four additional signs: low positive relative accommodation $(\leq 1.25$ D), failing monocular accommodative facility with minus lenses of $\pm 2 \mathrm{D}$ flipper lenses, failing binocular accommodative facility with minus lenses of $\pm 2 \mathrm{D}$ lenses, and a value of monocular estimated method (MEM) dynamic retinoscopy $\geq 1$ D. Similarly, Porcar and Martínez-Palomera ${ }^{10}$ used several signs, although they did not specify how many signs were necessary to make an accurate diagnosis. They focused the diagnosis on the signs of a reduced amplitude of accommodation ( $2 \mathrm{D}$ less than minimum), a value of positive relative accommodation $\leq 1.25 \mathrm{D}$, difficulty clearing with negative lenses in both monocular and binocular accommodative facility with $\pm 2 \mathrm{D}$ flipper lenses, a high MEM finding $(\geq+0.75 \mathrm{D})$, and a value of fused cross-cylinder $\geq 1 \mathrm{D}$.

Recently, Lara et al. ${ }^{11}$ studied the prevalence of general binocular dysfunctions in a clinic population and reported the number of signs used for diagnosing the accommodative insufficiency. They established the necessity of two signs to be present: reduced amplitude of accommodation (at least $2 \mathrm{D}$ below minimum Hofstetter's calculation for age) and failing monocular accommodative facility with $-2 \mathrm{D}$ lenses $(\leq 6 \mathrm{cpm})$. Furthermore, two additional signs of the following three needed to be present: failing binocular accommodative facility with $-2 \mathrm{D}$ lenses $(\leq 3 \mathrm{cpm})$, a MEM finding greater than $+0.75 \mathrm{D}$, and a positive relative accommodation $\leq 1.25 \mathrm{D}$.

As can be observed, there is a lack of agreement concerning the number of clinical signs that combined with diminished accommodative amplitude must be used for classifying accommodative insufficiency and what their importance must be to give an accurate diagnosis. For that reason, we attempted to determine the most sensitive tests (together with accommodative amplitude) for classifying accommodative insufficiency. For this purpose, we analyzed the relationship between MEM dynamic retinoscopy, monocular and binocular accommodative facility, and positive relative accommodation with or without the presence of reduced amplitude of accommodation.

\section{METHODS}

From the symptomatic patients presented consecutively to an optometric clinic, we chose 328 subjects aged 13 to 35 years. All subjects gave their informed consent after having the nature of the tests to be performed explained to them, and they authorized the authors to apply the results obtained in this research. All subjects had normal ocular and systemic health and had at least 20/20 visual acuity with their best correction. None of the subjects wore contact lenses, and subjects with strabismus were excluded. We completed a visual examination including the following tests:

- Patient's history, reflecting the full range of symptoms presented by the patient. The most common reasons of presenting complaint were symptoms associated with reading or other close work, difficulty with near tasks, inability to concentrate and a loss of comprehension over time, ocular fatigue, asthenopia, headaches, blurred vision, eyestrain, diplopia, and sensitivity to light.

- Ocular health by means of ophthalmoscopy and biomicroscopy.

- Assessment of refractive error. Static retinoscopy was performed while the patient fixated a distant chart at $6 \mathrm{~m}$. A subjective examination was performed by means of monocular fogging method with cross-cylinder, followed by binocular balancing to a standard endpoint of maximum plus for best visual acuity (BVA). 
- Accommodative and binocular vision testing. With the results of the subjective refractive examination in place (BVA correction), we conducted an evaluation of accommodative and binocular vision including different tests ${ }^{12}$ : Assessment of direction and magnitude of the horizontal and vertical phoria was performed with the cover test and prism bar at $6 \mathrm{~m}$ and $40 \mathrm{~cm}$. AC/A ratio was measured by means of gradient and calculated methods. MEM dynamic retinoscopy ${ }^{13}$ was performed at $40 \mathrm{~cm}$ with the result of the subjective examination placed in a trial frame and using trial lenses. Positive and negative relative accommodations (PRA, NRA) were assessed while the patient was fixating the horizontal line of 20/20 letters at $40 \mathrm{~cm} .{ }^{12}$ Monocular and binocular accommodative facility (MAF, BAF) was conducted following the procedure of Zellers et al. ${ }^{14}$ We tested both accommodative facility at a distance of $40 \mathrm{~cm}$ using \pm 2 D flip lenses and a target with suppression control. Monocular accommodative amplitude (AA) was measured with the push-up method. We also measured the positive and negative fusional vergences at distance and near $(6 \mathrm{~m}$ and $40 \mathrm{~cm}$, respectively) and the nearpoint of convergence. Finally, we examined stereopsis with Wirt circles (Titmus stereopsis test) and fixation disparity with Wesson card.

To avoid the examiner bias for particular results of the tests, one author performed the visual examinations, and another author analyzed the patient data.

Based on the optometric literature ${ }^{1,3,5-11}$ that accommodative amplitude is the essential sign for diagnosing accommodative insufficiency, we selected patients who presented an amplitude of accommodation at least $2 \mathrm{D}$ below minimum age-appropriate amplitude according to Hofstetter's formula ${ }^{2}: 15-0.25 \times$ age. From this analysis, we obtained 41 patients who exhibited that condition. In addition, we consecutively selected data from 40 subjects (control group) who did not have any accommodative and/or binocular disorders and whose accommodative amplitudes were normal.

Because accommodative insufficiency is not only related to a low accommodative amplitude but also to signs such as high MEM dynamic retinoscopy, failing monocular and binocular accommodative facility, and low positive relative accommodation, ${ }^{8-11}$ we used these four signs to determine their specificity and sensitivity at detecting accommodative insufficiency in our sample of subjects with diminished accommodative amplitude.

For this analysis, it is necessary to define the "pass" criterion level for each of these tests, so we considered the mean and standard deviation values published by Scheiman and Wick ${ }^{12}$ to determine the cutoff of each sign (Table 2). According to the normal values proposed by these authors, two different types of criteria for establishing the cutoff can be defined. The first is using the limit determined by the standard deviation. As an example, the limit of MEM is $+0.75 \mathrm{D}$, so we can consider a result of $+1.00 \mathrm{D}$ or higher as failing the test and a value of $+0.75 \mathrm{D}$ or lower as passing the test. A similar assessment can be used for the other signs (Table 2). The second criterion uses the mean value as the cutoff, so the suspect values defined by the standard deviation imply failing the test. In the previous example, MEM values of $+0.50 \mathrm{D}$ or lower would be considered as passing the test, and results of $+0.75 \mathrm{D}$ or higher would be taken as failing the test.

Comparing the results of each criterion, it can be established which cutoff offers the best sensitivity and specificity values. Thus, with the results of the four tests studied for both groups of patients established in our study (41 subjects with diminished accommodative amplitude and 40 subjects with normal amplitude), we determined the sensitivity and specificity for each one of the signs, using the right eye results when the monocular tests were studied.

Specificity can be understood as the proportion of normal cases (in this paper, the subjects with normal accommodative amplitude) that are correctly identified by passing the test, which is calculated by dividing the number of true negatives (TN) by the sum of true negatives and false positives (FP): TN/(TN + FP). Sensitivity is the proportion of subjects with diminished accommodative amplitude who are correctly identified by failing the test. Sensitivity is calculated by dividing the number of true positives (TP) by the sum of the true positives and false negatives (FN): $\mathrm{TP} /(\mathrm{TP}+\mathrm{FN})$. In our case, positives are considered to be the group of 41 subjects with diminished accommodative amplitude. True positives are the number of subjects who did not pass the test and were in the group of low accommodative amplitude. False positives are the number of patient who did not pass the test but who were in the normal group.

Negatives are the group of 40 subjects who have normal accommodative amplitude and have no accommodative or binocular dysfunction (control group). True negatives are patients who passed the test and were in normal group, and false negatives are subjects who passed the test but were in the group with diminished accommodative amplitude.

\section{RESULTS}

Using the cutoff established by the standard deviation for each test studied, the specificity was very high for all tests analyzed (Table 3). These values indicate that there is a high probability that

\section{TABLE 2.}

Cut-off used to calculate specificity and sensitivity.

\begin{tabular}{lccc}
\hline Signs & Mean Value \pm SD & Failing the Test with SD & Failing the Test with Mean Value \\
\hline MEM $^{a}$ & $+0.50 \pm 0.25 \mathrm{D}$ & $\geq+1.00 \mathrm{D}$ & $\geq+0.75 \mathrm{D}$ \\
MAF & $11 \pm 5 \mathrm{cpm}$ & $\leq 6 \mathrm{cpm}($ with $-2.00 \mathrm{D})$ & $\leq 10 \mathrm{cpm}($ with $-2.00 \mathrm{D})$ \\
BAF & $8 \pm 5 \mathrm{cpm}$ & $\leq 3 \mathrm{cpm}($ with $-2.00 \mathrm{D})$ & $\leq 7 \mathrm{cpm}($ with $-2.00 \mathrm{D})$ \\
PRA & $2.37 \pm 1.00 \mathrm{D}$ & $\leq 1.25 \mathrm{D}$ & $\leq 2.25 \mathrm{D}$ \\
\hline
\end{tabular}

a MEM, monocular estimated method dynamic retinoscopy; MAF, monocular accommodative facility; BAF, binocular accommodative facility; PRA, positive relative accommodation. 
a subject who passes each test could be considered as a patient with normal accommodative amplitude. If the cutoff defined by the mean value of each test is used (Table 2), the specificity does not suffer, except for MEM dynamic retinoscopy, which diminishes to a value of 0.48 . This suggests that for obtaining an adequate value of specificity, the standard deviation value should be used as the cutoff for MEM retinoscopy.

To determine the sensitivity, the 41 patients with diminished amplitude of accommodation must be studied. Data for these subjects are represented in Table 4, where it has been specified when each patient passed or failed the test as well as whether the result was within suspect values defined by the standard deviation. Furthermore, to identify patients who could have a vergence problem, we have indicated those who had a significant heterophoria at near ( $>2 \Delta$ of esophoria or $>6 \Delta$ of exophoria). ${ }^{12}$

When the standard deviation value was used as a cutoff, the sensitivity of the four tests used for diagnosing accommodative insufficiency was not very high (Table 5). Therefore, it cannot be assured that a patient who fails these tests will have diminished amplitude of accommodation. Although these sensitivity values are not particularly encouraging, it is interesting to compare the results of each test. As can be observed, the higher sensitivity values were associated with high MEM dynamic retinoscopy values and failing the monocular accommodative facility with negative lenses. The binocular accommodative facility and positive relative accommodation showed lower values of sensitivity.

If the mean value was used as cutoff, the sensitivity increased due to a greater number of true positives. As can be observed in Table 5 , this was the case for all tests, resulting in greatest sensitivity for MEM retinoscopy and for positive relative accommodation, whereas monocular and binocular accommodative facility showed similar values. However, the increase in sensitivity was not the same for all signs: PRA increased from 0.27 to 0.49 , whereas the MAF hardly modified its sensitivity (from 14 to 16 true positives). These results suggest that changing the cutoff modifies the sensitivity, except in the case of monocular accommodative facility.

As we have shown, the sensitivity values were never high, so it can be deduced that most of the 41 patients with diminished accommodative amplitude were not related to accommodative insufficiency because they did not present anomalous values in tests that would be affected by this accommodative anomaly. To separate the patients who simply had an accommodative disorder from the original 41 subjects, we eliminated subjects who presented a significant heterophoria at near distance $(>2 \Delta$ esophoria and $>6$ $\Delta$ exophoria). Thus, the sample of patients was reduced to 26 patients for whom we calculated the sensitivity for the two established cutoffs.
Table 6 shows the sensitivity values for these 26 patients. Using the standard deviation criterion, the highest sensitivity was for MAF, followed by MEM and PRA, although again high values are not reached. When the mean value was used as the cutoff, the sensitivity increased, with MAF, MEM, and PRA reaching 0.58 . Again, PRA was the sign that had the highest increase of sensitivity, whereas MAF changed little.

Similar to the original analysis, most of the patients without significant heterophorias at near could not be considered to have accommodative insufficiency because in no cases were the sensitivity values high.

\section{DISCUSSION}

According to our results, the four signs studied for diagnosing accommodative insufficiency have a high specificity for both cutoff criteria, with the exception of MEM dynamic retinoscopy when the mean value is used as the cutoff. These results indicate that a very high percentage of patients who reach the mean values of PRA, MAF, and BAF will not have diminished accommodative amplitude. In the case of MEM dynamic retinoscopy, it is necessary to use the standard deviation as the cutoff to maintain a high level of specificity. However, these specificity values are of marginal clinical interest: normal values of each test are not associated with an anomaly of the accommodative system.

The sensitivity analysis offers more interesting results. The sensitivity values were never high, so there was no sign strongly associated with the presence of diminished amplitude of accommodation. However, most clinicians rely on accommodative amplitude as a "gold standard" for diagnosing the accommodative insufficiency. Our results suggest the lack of this gold standard, indicating a difficulty for diagnosing this accommodative anomaly as other signs have been taken into account. Nonetheless, a reduction in the accommodative amplitude cannot be used as the only sign for diagnosing accommodative insufficiency if one assumes that in this accommodative anomaly other signs must be affected.

When analyzing the sensitivity of the four signs studied, the values of BAF were the lowest for both cutoffs used and for both samples studied. This can be explained by the fact that binocular accommodative facility is a binocular test, and failing it does not imply necessarily the presence of a monocular problem. ${ }^{15}$

Concerning the PRA, its sensitivity values were very variable depending on the cutoff considered. When the standard deviation value was used, the sensitivity offered low values in both samples but increased considerably when the mean value was used as the cutoff. In fact, the PRA was the sign with the greatest increase of true positives when the cutoff was changed. This finding suggests

TABLE 3.

Specificity for the sample of 40 patients with normal accommodative amplitude.

\begin{tabular}{|c|c|c|c|c|}
\hline Test Failure & MEM $^{a}$ & MAF & BAF & PRA \\
\hline Standard deviation & $\begin{array}{c}0.88 \\
35 /(35+5)\end{array}$ & $\begin{array}{c}1 \\
40 /(40+0)\end{array}$ & $\begin{array}{c}0.93 \\
37 /(37+3)\end{array}$ & $\begin{array}{c}1 \\
40 /(40+0)\end{array}$ \\
\hline Mean value & $\begin{array}{c}0.48 \\
19 /(19+21)\end{array}$ & $\begin{array}{c}1 \\
40 /(40+0)\end{array}$ & $\begin{array}{c}0.90 \\
36 /(36+4)\end{array}$ & $\begin{array}{c}0.93 \\
37 /(37+3)\end{array}$ \\
\hline
\end{tabular}

a MEM, monocular estimated method dynamic retinoscopy; MAF, monocular accommodative facility; BAF, binocular accommodative facility; PRA, positive relative accommodation. 
618 Accommodative Insufficiency-Cacho et al.

TABLE 4.

Data for the 41 subjects with diminished amplitude of accommodation.

\begin{tabular}{|c|c|c|c|c|c|}
\hline Subject & $\mathrm{MEM}^{a}$ & PRA & MAF & BAF & Phoria \\
\hline 1 & & $\bigcirc^{b}$ & & & Exo \\
\hline 2 & 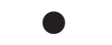 & & 0 & 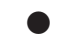 & \\
\hline 3 & & & & & \\
\hline 4 & & $\bigcirc$ & & & \\
\hline 5 & 0 & 0 & & 0 & Eso \\
\hline 6 & 0 & 0 & 0 & 0 & \\
\hline 7 & & & & & Exo \\
\hline 8 & 0 & 0 & ○ & - & \\
\hline 9 & 0 & & & & Eso \\
\hline 10 & $\bigcirc$ & & & & Exo \\
\hline 11 & 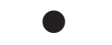 & 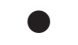 & 0 & $\bigcirc$ & \\
\hline 12 & & & & & Exo \\
\hline 13 & 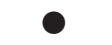 & 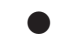 & 0 & $\bigcirc$ & \\
\hline 14 & & & & & \\
\hline 15 & $\bigcirc$ & & & & \\
\hline 16 & & & & & \\
\hline 17 & 0 & & & $\bigcirc$ & Eso \\
\hline 18 & & & & & \\
\hline 19 & 0 & & $\bigcirc$ & & Eso \\
\hline 20 & $\bigcirc$ & $\bigcirc$ & & & \\
\hline 21 & & & 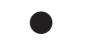 & 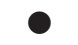 & \\
\hline 22 & & & & & \\
\hline 23 & 0 & 0 & 0 & 0 & \\
\hline 24 & 0 & 0 & 0 & & \\
\hline 25 & 0 & 0 & 0 & 0 & \\
\hline 26 & & & $\bigcirc$ & & \\
\hline 27 & $\bigcirc$ & 0 & & & Exo \\
\hline 28 & & 0 & & & Eso \\
\hline 29 & & & & & \\
\hline 30 & 0 & 0 & 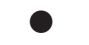 & $\bigcirc$ & \\
\hline 31 & & & & & Eso \\
\hline 32 & & & & 0 & Eso \\
\hline 33 & $\bigcirc$ & & & & \\
\hline 34 & 0 & 0 & & & Eso \\
\hline 35 & 0 & 0 & 0 & & \\
\hline 36 & 0 & 0 & 0 & 0 & \\
\hline 37 & & 0 & & & \\
\hline 38 & 0 & & & 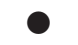 & Eso \\
\hline 39 & & 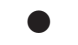 & 0 & 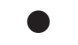 & \\
\hline 40 & & & & & Eso \\
\hline 41 & 0 & 0 & 0 & & \\
\hline
\end{tabular}

a MEM, monocular estimated method dynamic retinoscopy; PRA, positive relative accommodation; MAF, monocular accommodative facility; BAF, binocular accommodative facility; Exo, exophoria; Eso, esophoria.

${ }^{b}(\bigcirc)$ represents failing the test, and $(\bigcirc)$ represents suspect values.

that some patients can have a suspect value of PRA that is related to accommodative insufficiency. Nonetheless, using only this sign for the diagnosis of accommodative insufficiency would not be adequate.

MEM dynamic retinoscopy always presents one of the highest values of sensitivity compared with the other signs. However, the number of true positives in each of the two samples were very different: 18 and 12 with the standard deviation as the cutoff and 23 and 15 using the mean value for 41 and 26 patients, respectively. This variability is explained due to the fact that having a high MEM finding is not only related to accommodative insufficiency but to convergence excess, ${ }^{9-11}$ so this sign could not be considered alone for diagnosing accommodative insufficiency.
The monocular accommodative facility reaches relatively high sensitivity values compared with the other signs. Only in the sample of 41 patients using the mean value as the cutoff can the sensitivity be considered low. Contrary to what happened with MEM dynamic retinoscopy, the number of patients who failed the MAF was very similar for the four situations studied: for each of the two cutoff used and for both samples of patients considered (41 and 26 subjects). There were 14 patients who failed the test of MAF and only two subjects who presented suspect values (Table 4). Of these two patients, one of them had a high heterophoria at near. This suggests that failing MAF is not related to significant heterophoria at near. In fact, failing monocular accommodative 
TABLE 5.

Sensitivity results for the sample of 41 patients using different failure criteria for each sign.

\begin{tabular}{lcccc}
\hline \multicolumn{1}{c}{ Test Failure } & MEM $^{a}$ & MAF & BAF & PRA \\
\hline Standard deviation & 0.44 & 0.34 & 0.27 & 0.27 \\
Mean value & $18 /(18+23)$ & $14 /(14+27)$ & $11 /(11+30)$ & 0.37 \\
& 0.56 & 0.39 & $11 /(11+30)$ & 0.49 \\
& $23 /(23+18)$ & $16 /(16+25)$ & $20 /(20+21)$ \\
\hline
\end{tabular}

${ }^{a}$ MEM, monocular estimated method dynamic retinoscopy; MAF, monocular accommodative facility; BAF, binocular accommodative facility; PRA, positive relative accommodation.

TABLE 6.

Sensitivity results for the sample of 26 patients using different failure criteria for each sign.

\begin{tabular}{|c|c|c|c|c|}
\hline Test Failure & MEM $^{a}$ & MAF & BAF & PRA \\
\hline \multirow[t]{2}{*}{ Standard deviation } & 0.46 & 0.54 & 0.31 & 0.38 \\
\hline & $12 /(12+14)$ & $14 /(14+12)$ & $8 /(8+18)$ & $10 /(10+16)$ \\
\hline \multirow[t]{2}{*}{ Mean value } & 0.58 & 0.58 & 0.42 & 0.58 \\
\hline & $15 /(15+11)$ & $15 /(15+11)$ & $11 /(11+15)$ & $15 /(15+11)$ \\
\hline
\end{tabular}

${ }^{a}$ MEM, monocular estimated method dynamic retinoscopy; MAF, monocular accommodative facility; BAF, binocular accommodative facility; PRA, positive relative accommodation.

facility with $-2 \mathrm{D}$ lenses is only associated with the presence of the accommodative insufficiency and not with any other accommodative or binocular disorders. ${ }^{9-11}$

All of these results suggest that of the four signs studied, the MAF is the sign that is most closely related to accommodative insufficiency. For that reason we propose to use diminished accommodative amplitude together with failing with minus lenses in the monocular accommodative facility to diagnose accommodative insufficiency. To evaluate this proposal, we compared the diagnosis of accommodative insufficiency using this criterion with the criteria used by Hokoda, ${ }^{7}$ Scheiman et al., ${ }^{9}$ and Lara et al. ${ }^{11}$; these are the only reports in which it is clearly established how they diagnose this accommodative anomaly using several signs.

Using the criteria of Hokoda $^{7}$ (AA diminished plus PRA $\leq 1.25$ D), 11 of our patients would have accommodative insufficiency. According to the criteria proposed by Scheiman et al. ${ }^{9}$ (AA diminished plus two of four additional signs), 17 of our patients would have accommodative insufficiency. Using the criteria of Lara et al. ${ }^{11}$ (AA diminished plus MAF $\leq 6 \mathrm{cpm}$ with $-2 \mathrm{D}$ lenses plus two of three additional signs), 13 of our patients who would have accommodative insufficiency. With our proposed criterion (AA diminished plus MAF $\leq 6 \mathrm{cpm}$ with $-2 \mathrm{D}$ lenses), 14 patients would have accommodative insufficiency.

As can be observed, our results show that for accommodative insufficiency, the number of patients diagnosed with this anomaly depends on the sign used and not necessarily on the number of signs used for its diagnosis. However, these results do not agree with what occurs in some general binocular disorders in which it is shown that when using more signs for diagnosing the anomaly, the number of diagnosed patients decreases. ${ }^{16}$

Finally, if patients with significant heterophoria at near are not considered (Table 4), the number of subjects diagnosed with accommodative insufficiency would be 10 using the criteria of Hokoda, ${ }^{7} 13$ using the criteria of Lara et al., ${ }^{11} 14$ using the criteria suggested here, and also the same 14 patients following the criteria of Scheiman et al. ${ }^{9}$

All these results suggest that the presence of the accommodative insufficiency is related to several signs that are affected at the same time because the number of patients diagnosed hardly varied when electing two, three, or four different signs. That is, when accommodative insufficiency really exists, the majority of the four signs related to this anomaly will be affected, so it would not be necessary to use all of them in the diagnosis. For that reason, we consider that using only the sign of failing monocular accommodative facility with minus lenses together with diminished accommodative amplitude, we would be able to diagnose patients with accommodative insufficiency. In any case, it is clear that the signs must be compared with the symptoms of the patient so a good diagnosis can take into account both symptoms and the results of the accommodative tests. In our case, the 14 patients diagnosed with accommodative insufficiency presented symptoms associated with this anomaly, mainly difficulty and discomfort associated with near tasks.

\section{CONCLUSIONS}

The specificity obtained is high for all the tests analyzed, so a patient who reaches normal values for MEM dynamic retinoscopy, monocular and binocular accommodative facility, and positive relative accommodation will not be related to accommodative insufficiency.

Of the four signs studied, none of them reached high values of sensitivity, so they cannot be directly associated with a diminished amplitude of accommodation. If accommodative insufficiency is considered as a disorder in which several signs are affected, it is not appropriate to use the accommodative amplitude as the only sign for diagnosing it.

According to the sensitivity results, with the two cutoff values 
established in our study and for both samples considered, failing monocular accommodative facility with $-2 \mathrm{D}$ lenses seems to be the sign most associated with accommodative insufficiency. Thus, we propose using MAF together with diminished accommodative amplitude for diagnosing this accommodative anomaly. Using this suggested criterion, the number of patients diagnosed with accommodative insufficiency in our sample of patients is similar to the number of subjects obtained with the criteria proposed by other authors.

\section{ACKNOWLEDGMENTS}

Portions of this manuscript were previously presented at the Sixth International Meeting of the American Academy of Optometry (Madrid, Spain, April 7-9, 2000).

Received November 20, 2001; revision received March, 22, 2002.

\section{REFERENCES}

1. Daum KM. Accommodative dysfunction. Doc Ophthalmol 1983; 55:177-98.

2. Hofstetter HW. Useful age-amplitude formula. Optom World 1950; 38:42-5.

3. Matsuo T, Ohtsuki H. Follow-up results of a combination of accommodation and convergence insufficiency in school-age children and adolescents. Graefes Arch Clin Exp Ophthalmol 1992;230:166-70.

4. Duane KM. Studies in monocular and binocular accommodation with their clinical application. Am J Ophthalmol 1922;5:565-77.

5. Russell GE, Wick B. A prospective study of treatment of accommodative insufficiency. Optom Vis Sci 1993;70:131-5.

6. Dwyer P, Wick B. The influence of refractive correction upon disorders of vergence and accommodation. Optom Vis Sci 1995;72: 224-32.

7. Hokoda SC. General binocular dysfunctions in an urban optometry clinic. J Am Optom Assoc 1985;56:560-2.

8. Rouse MW, Borsting E, Hyman L, Hussein M, Cotter SA, Flynn M,
Scheiman M, Gallaway M, De Land PN, The Convergence Insufficiency and Reading Study (CIRS) Group. Frequency of convergence insufficiency among fifth and sixth graders. Optom Vis Sci 1999;76: 643-9.

9. Scheiman M, Gallaway M, Coulter R, Reinstein F, Ciner E, Herzberg C, Parisi M. Prevalence of vision and ocular disease conditions in a clinical pediatric population. J Am Optom Assoc 1996;67:193-202.

10. Porcar E, Martinez-Palomera A. Prevalence of general binocular dysfunctions in a population of university students. Optom Vis Sci 1997; 74:111-3.

11. Lara F, Cacho P, García A, Megías R. General binocular disorders: prevalence in a clinic population. Ophthalmic Physiol Opt 2001;21: $70-4$.

12. Scheiman M, Wick B. Clinical Management of Binocular Vision: Heterophoric, Accommodative and Eye Movement Disorders. Philadelphia: JB Lippincott, 1994:3-31.

13. Rouse MW, London R, Allen DC. An evaluation of the monocular estimate method of dynamic retinoscopy. Am J Optom Physiol Opt 1982;59:234-9.

14. Zellers JA, Alpert TL, Rouse MW. A review of the literature and a normative study of accommodative facility. J Am Optom Assoc 1984; 55:31-7.

15. García A, Cacho P, Lara F, Megías R. The relation between accommodative facility and general binocular dysfunction. Ophthalmic Physiol Opt 2000;20:98-104.

16. Rouse MW, Hyman L, Hussein M, Solan H. Frequency of convergence insufficiency in optometry clinic settings. Convergence Insufficiency and Reading Study (CIRS) Group. Optom Vis Sci 1998;75: 88-96.

Pilar Cacho Martínez

Departamento Interuniversitario de Óptica Apartado 99. Universidad de Alicante 03080 Alicante, Spain e-mail: cacho@ua.es 